\title{
Changes in the Metastability of the Midlatitude Southern Hemisphere Circulation and the Utility of Nonstationary Cluster Analysis and Split-Flow Blocking Indices as Diagnostic Tools
}

\author{
TEREnCE J. O'Kane And James S. Risbey \\ CSIRO Marine and Atmospheric Research, Hobart, Tasmania, Australia \\ CHRISTIAN FRANZKE \\ British Antarctic Survey, Cambridge, United Kingdom \\ ILLIA HORENKO \\ Universita della Svizzera Italiana, Lugano, Switzerland \\ DIDIER P. MONSELESAN \\ CSIRO Marine and Atmospheric Research, Hobart, Tasmania, Australia
}

(Manuscript received 24 January 2012, in final form 6 September 2012)

\begin{abstract}
Changes in the metastability of the Southern Hemisphere 500-hPa circulation are examined using both cluster analysis techniques and split-flow blocking indices. The cluster methodology is a purely data-driven approach for parameterization whereby a multiscale approximation to nonstationary dynamical processes is achieved through optimal sequences of locally stationary fast vector autoregressive factor (VARX) processes and some slow (or persistent) hidden process switching between them. Comparison is made with blocking indices commonly used in weather forecasting and climate analysis to identify dynamically relevant metastable regimes in the 500-hPa circulation in both reanalysis and Atmospheric Model Intercomparison Project (AMIP) datasets. The analysis characterizes the metastable regime in both reanalysis and model datasets prior to 1978 as positive and negative phases of a hemispheric midlatitude blocking state with the southern annular mode (SAM) associated with a transition state. Post-1978, the SAM emerges as a true metastable state replacing the negative phase of the hemispheric blocking pattern. The hidden state frequency of occurrences exhibits strong trends. The blocking pattern dominates in the early 1980s, and then gradually decreases. There is a corresponding increase in the SAM frequency of occurrence. This trend is largely evident in the reanalysis summer and spring but was not evident in the AMIP dataset. Further comparison with the splitflow blocking indices reveals a superficial correspondence between the cluster hidden state frequency of occurrences and split-flow indices. Examination of composite states shows that the blocking indices capture splitting of the zonal flow whereas the cluster composites reflect coherent block formation. Differences in blocking climatologies from the respective methods are discussed.
\end{abstract}

\section{Introduction}

The formation of quasi-stationary high pressure systems in the atmospheric midlatitudes is often referred to as "blocking." The formation of a coherent blocking

\footnotetext{
Corresponding author address: Terence O'Kane, CSIRO Marine and Atmospheric Research, Castray Esplanade, Hobart TAS 7001, Australia.

E-mail: terence.okane@csiro.au
}

structure is necessarily associated with a reduction in the strength of the zonal circulation and a corresponding enhancement of the meridional motion. In the Southern Hemisphere (SH), midlatitude blocks may persist on a time scale on the order of a week or longer. Charney and DeVore (1979) first proposed that multiple equilibria may be a possible mechanism for blocking events. Their hypothesis states that the observed multiple weather regimes are quasi-stationary or metastable states and that instability mechanisms are responsible for initiating 
transitions between said states. In this regard the blocked and unblocked weather patterns are examples of such regimes. The physical mechanism that generates multiple equilibria proposed by Charney and DeVore can be summarized as follows: strong zonal (eastward) jets in the midlatitudes and the Coriolis effect create meridional temperature gradients, topography generates Rossby waves in turn, creating drag on the flow and pushing it westward. Under suitable conditions and for a particular value of the zonal wind the waves might exhibit a resonant response, causing the large-scale flow to become locked near the resonant wind value. Thus, for particular values of zonal forcing, dissipation, or topographic height, the flow will settle into either the state with winds near the zonal forcing value or to one with winds near the resonant wind value, depending on the initial conditions.

Zidikheri et al. (2007) showed that a severely truncated regional barotropic beta-plane model of the atmosphere with only topographically excited waves in a background flow could possesses multiple equilibria for a wide range of physically plausible parameters. They further showed that multiple equilibria exist in a hierarchy of models of increasing complexity by the addition of extra modes, more realistic topographic distribution, and zonal jet structures. ${ }^{1}$ By introducing midlatitudinal jets into their (barotropic) model, they found that the zonal jet structure in global baroclinic models helps to confine topographic Rossby waves within a given latitudinal band and that this can lead to resonance without the excessive winds needed in a simple global barotropic scenario. They demonstrated that resonance is a crucial condition for the existence of multiple equilibria. Frederiksen $(1982,1983)$ later disentangled the respective roles of baroclinic and barotropic instability in block formation, maturation, and decay. He demonstrated the crucial role of baroclinic instability in the early stages of the development of patterns (growing error structures) resembling those observed during blocking and that barotropic instabilities were more important for the persistence of the mature blocking stage.

Branstator and Berner (2005) and Berner and Branstator (2007) showed that, even after low-frequency time filtering, the probability distribution functions (PDFs) of the planetary waves in long integrations of numerical general circulation models are nearly Gaussian and without any multiple extrema. This is despite highly nonGaussian PDF and multiple extrema found in the lowfrequency planetary waves of the statistically processed

\footnotetext{
${ }^{1}$ For a more complete discussion of the role of finite resolution in influencing regime behavior, see Zidikheri et al. (2007).
}

observational record (Cheng and Wallace 1993; Corti et al. 1999). Majda et al. (2006) examined this controversy using a simple 57-mode paradigm model for the angular momentum of the atmosphere whose low-order truncated model is exactly the 3D Charney-DeVore model without dissipation and forcing. Through hidden Markov model (HMM) analysis of the time series of suitable lowfrequency planetary waves they elucidated how statistically significant metastable regime transitions occur between blocked and zonal statistical states despite nearly Gaussian behavior through the critical role of turbulent backscatter onto the three-dimensional subspace of low-frequency modes.

Using statistical dynamical closure methods O'Kane and Frederiksen (2008) showed the importance of the cumulative contribution of non-Gaussian terms to the evolved error tendency as well as the role of the offdiagonal covariances in the growth of errors during a rapid regime transition associated with the formation of a block over the Gulf of Alaska in November 1979. Frederiksen et al. (2012) review the literature and recent advances in stochastic subgrid modeling; Frederiksen and O'Kane (2008) review recent developments in nonequilibrium statistical dynamical closure theory, its application to subgrid-scale modeling, and role in midlatitude regime transitions.

Tibaldi (1993) first proposed that the so-called splitflow blocking indices might be used as diagnostic tools for assessing global climate models. Using a modified version of the Lejenas and Oakland objective blocking index (Tibaldi and Molteni 1990; Tibaldi et al. 1994) they assessed 7 years of analyses and forecasts from the European Centre for Medium-Range Weather Forecasting (ECMWF) operational archives. They found only one preferred region for blocking in the $\mathrm{SH}$, around $180^{\circ}$ longitude, corresponding to the Australian blocking region and that blocking in the $\mathrm{SH}$ was considerably more difficult to characterize than in the Northern Hemisphere (NH). Pook and Gibson (1999) comprehensively review the development of blocking indices specific to the $\mathrm{SH}$ as used in operational weather prediction at the Australian Bureau of Meteorology. They note that there are three well-recognized $\mathrm{SH}$ blocking regions located to the east or southeast of the continents along latitude $45^{\circ} \mathrm{S}$ and that the Australian block (including the Tasman Sea and southwestern Pacific) is the most active. Development of a SH blocking index by the Extended Forecast Section of the Australian Bureau of Meteorology culminated in the work of Wright (1994). As blocking plays a critical role in determining precipitation in southern Australia, correct identification of such events is of great importance (Risbey et al. 2009). 
In the NH quasi-stationary blocking anticyclones split the zonal flow, causing the usual east-west propagation of migratory cyclones and anticyclones to be deflected along either a more northward or southward path. As blocks typically last for about a week or longer, this will certainly influence regional weather over periods longer than the typical life span of synoptic-scale cyclones. An early attempt at explaining changes in the frequency of occurrence of natural atmospheric circulation regimes in the $\mathrm{NH}$ using stationary cluster analysis can be found in Corti et al. (1999). More recently, Franzke et al. (2009) applied a clustering method to systematically identify metastable atmospheric regimes in high-dimensional datasets generated by a barotropic model and an atmospheric general circulation model (GCM). They employed a finite element clustering approach that decomposes the phase space into overlapping clusters while simultaneously estimating the most likely switching sequence among the clusters. The parameters of the clustering and switching are estimated by a finite element approach developed by Horenko (2009, 2010a, 2011). The switching among the clusters can be described by a Markov transition matrix (Majda et al. 2006; Franzke et al. 2008, 2009), while metastable regime behavior can be assessed by inspecting the eigenspectrum of the associated transition probability matrix. In the current paper we focus on the finite element, bounded variation, vector autoregressive factor (FEM-BV-VARX) method from Horenko $(2010 \mathrm{~b}, \mathrm{c})$ in combination with the Akaike information criterion (AIC) to decide the optimal cluster state as described in section 2.

In an analysis of $\mathrm{NH}$ data from the National Center for Atmospheric Research Community Climate Model 0 (CCM0), Franzke et al. (2009) identified spatial structures of seven metastable regimes corresponding to, among others, both phases of the northern annular mode and Pacific blocking. They showed that these regimes were maintained predominantly by transient eddy fluxes of low-pass filtered anomalies and demonstrated how the dynamical description of the slow process switching between the regimes can be acquired from the analysis results. Horenko (2010c) recently compared a NH blocking index to cluster affiliation sequences. He compared the Lejenas-Oakland blocking index to cluster affiliations for 40-yr ECMWF Re-Analysis (ERA-40) geopotential data in Europe for the period 1958-2003, noting good qualitative agreement. However, a detailed comparison of the relative merits of split-flow blocking indices to cluster analysis has not yet been performed and, in particular, for the SH.

The purpose of this article is to assess changes to the metastability of the $\mathrm{SH}$ atmospheric circulation and to assess the relative merits of operational split-flow blocking indices (Tibaldi et al. 1994; Wright 1994) as compared to one based on transition sequences from the finite element clustering (Horenko 2009, 2010a,c). We are also interested in comparing reanalysis data, wherein systematic changes to the $\mathrm{SH}$ circulation over the last 40 years are presumed due to changes in the radiative forcing and to AMIP model data, which presume climatological radiative forcing. In section 2 we outline the clustering method, including the information criteria used to determine the optimal parameter values, and the operational split-flow blocking indices. In section 3 we describe the reanalysis and GCM model datasets to be considered. Results and discussion and summary and conclusions are presented in sections 4 and 5 , respectively.

\section{Methodology}

In this section we describe the finite-element clustering method for the identification of regime states in high-dimensional non-Gaussian and non-Markovian geophysical datasets (Horenko 2009, 2010b). In an earlier study of $\mathrm{NH}$ atmospheric regimes Franzke et al. (2009) employed the FEM-H1-EOF clustering method from Horenko (2010a), an H1-regularized FEM clustering based on EOF-distance metric $\left\|x(t)-T(t) T(t)^{*} x(t)\right\|$, and homogenous (i.e., stationary) spectral Markov criterion to decide the optimal number of clusters $K$. In Franzke et al. (2009) it was for the first time demonstrated that the FEM-H1-EOF methodology (Horenko 2010a) can be successfully applied to the identification of relevant persistent atmospheric circulation regimes. A characteristic property of the FEM-H1-EOF is that it considers the persistent dynamical regimes to be manifested through the (slow) temporal change of the lowdimensional linear EOF manifold $T(t)$ of dimensionality $d$. In the context of FEM-H1-EOF, this is achieved via a minimization of a geometrical distance between the data and the manifold, subject to the H1-regularization, guaranteeing that the resulting manifold is slow.

In the current paper we employ the FEM-BV-VARX method from Horenko (2010c): a BV-regularized FEM clustering based on VARX-distance metric $\left\|x(t)-\mu(t)-\sum_{i=1}^{m} A_{i}(t)^{*} x(t-i \tau)-B(t) u_{t}\right\|$ with the Akaike information criterion (AIC) to decide the optimal cluster state(s) $K$. As was demonstrated in Horenko (2010c), this method can be successfully applied to identify blocking events in the $\mathrm{NH}$ from the slow temporal changes of the statistical model parameters describing the dynamical interactions of the geopotential pressure values with some global atmospheric factors [e.g., North Atlantic Oscillation (NAO), $\mathrm{CO}_{2}$ concentration, and solar activity]. In this approach an empirical 
orthogonal function (EOF) decomposition is performed prior to clustering to reduce the dimensionality but is not formally a part of the procedure. Therefore, in contrast with the FEM clustering based on the EOF metrics, one cannot speak of a slow manifold here since the EOF manifold and the manifold for which the VARX dynamics is running are the same.

The FEM-BV-VARX algorithm does not simultaneously estimate a transition matrix for the cluster or metastable state evolution. Thus, if one were to fit a Markov matrix, assuming stationarity, the transition matrix and Markovianity would have to be computed and established a posteriori using a generator algorithm (see Crommelin and Vanden-Eijnden 2006; Franzke et al. 2009). In general, rather than calculate the transition matrix to determine the number of metastable states, we use the AIC and make no assumption of stationarity.

The FEM-BV-VARX (Horenko 2010c) method used simultaneously estimates the clusters (corresponding to regimes) and the most likely metastable state transitions between the clusters through the minimization of an average clustering functional $\mathbf{L}$ of a given time series $x_{t}$. This approach assumes that the dynamics of the observed variable of interest $x_{t}$ is influenced by the previous $m$ time-lagged values of the same variable (to describe the memory effects), some set of explicitly observed external factors $u_{t}$, and an unobservable (hidden) impact variable associated with regime transitions that strongly influences the observed variable.

\section{a. FEM-BV-VARX methodology}

Implicit in the FEM-BV-VARX approach is to assume that the dynamics may be approximated by a stochastic model of the following general form:

$\mathbf{x}_{t}=\mu_{t}+\mathbf{A}_{q}(t) \phi_{1}\left(x_{t-\tau}, \ldots, x_{t-m \tau}\right)+\mathbf{B}(t) \phi_{2}\left(u_{t}\right)+\mathbf{C}(t) \epsilon_{t}$,

where $\boldsymbol{\Theta}(t)=[\mu(t), \mathbf{A}(t), \mathbf{B}(t), \mathbf{C}(t)]$ is the vector of timedependent model parameters, $\epsilon_{t}$ is a stochastic variable describing how noisy the process is, $\phi_{1}\left(x_{t-\tau}, \ldots, x_{t-m \tau}\right)$ is some (in general, nonlinear function connecting the previous observations $\left.x_{t-\tau}, \ldots, x_{t-m \tau}\right), \phi_{2}\left(u_{t}\right)$ is an external factor function, and $\mathbf{C}(t)$ couples the unobserved scales (modeled as a statistically independent and identically distributed noise process with zero expectation) to the analyzed time series. As demonstrated in Horenko (2010a, 2011), time dependence of the model parameters $\boldsymbol{\Theta}$ is induced by the influence of the unresolved scales and leads to a nonstationary regime transition behavior in many realistic systems.
The FEM-BV-VARX method then tries to identify the time-dependent optimal parameters $\boldsymbol{\Theta}(t)$ as a convex linear combination of time-independent parameters $\theta_{i}, i=1, \ldots, K$ with time-dependent linear combination coefficients $\gamma_{i}(t)$. In context of the FEM-BV-VARX, functions $\gamma_{i}(t)$ can be interpreted as probabilities that the given observation $x_{t}$ at time $t$ can be best explained by a VARX model with constant model parameters $\theta_{i}$. Thus, for an a priori given number $K$ of clusters, fixed given time series $x_{t}$ and $u_{t}$, and fixed maximal time lag $m$, the FEM-BV-VARX methodology minimizes the distance of the actual trajectory [in an appropriate metric $\left.g\left(x_{t}, u_{t}, \theta_{i}\right)\right]$ to one of the $\mathrm{K}$ model clusters at time $t$. This means that we are looking simultaneously for the cluster locations [defined by the cluster probabilities $\left.\gamma_{i}(t), i=1, \ldots, K\right]$ and the time evolution of the system in the space spanned by the model clusters defined by the parameters $\theta_{i}$.

The method considers the clustering of possibly nonstationary multidimensional data $x_{t} \in R^{d}$ as a minimization problem:

$$
\mathbf{L}(\boldsymbol{\Theta}, \boldsymbol{\Gamma})=\sum_{t=0}^{T} \sum_{i=1}^{K} \gamma_{i}(t) g\left(x_{t}, u_{t}, \theta_{i}\right) \rightarrow \frac{\min }{\boldsymbol{\Gamma}(\mathbf{t}), \boldsymbol{\Theta}},
$$

subject to constraints

$$
\sum_{i=1}^{K} \gamma_{i}(t)=1, \quad \forall \quad t \in[0, T]
$$

and

$$
\gamma_{i}(t) \geq 0, \quad \forall \quad t \in[0, T], \quad i=1, \ldots, K,
$$

where we want to minimize the object $\mathbf{L}$. The corresponding cluster distance functional characterizes how well a given observation $x_{t}$ at time $t$ is described by a given model $i$ with parameters $\theta_{i}$ [see Horenko (2010a) for more details]. As demonstrated in Horenko (2009, 2010a), one can incorporate additional information into the optimization, such as some persistency assumptions of functions in space $[\boldsymbol{\Gamma}(\cdot)]$, and then apply a finite Galerkin time discretization of this infinite-dimensional Hilbert space. For example, for a given observation time series one can impose the constraint of the limited (i.e., bounded) temporal variation of the underlying statistical parameters $\Theta(t)$.

The optimization problem is now solved by a finite element approach [see Horenko (2009, 2010a,b) for more information and a detailed description of the algorithm] using principal components of the EOFs as described in Horenko (2008). The persistency constraint $C$ bounds the persistency of the function $\gamma_{i}$ via the norm 


$$
\begin{aligned}
\left|\gamma_{i}\right|_{\mathrm{BV}(0, T)} & =\sum_{t=0}^{T-1}\left|\gamma_{i}(t+1)-\gamma_{i}(t)\right|=\left\|\mathbf{D} \gamma_{i 1}^{\dagger}\right\| \leq C, \\
\mathbf{D} & =\left[\begin{array}{cccc}
-1 & 1 & \cdots & 0 \\
0 & -1 & \cdots & 0 \\
\vdots & & \ddots & \vdots \\
0 & \cdots & -1 & 1
\end{array}\right]
\end{aligned}
$$

where $i=1, \ldots, K$, the scalar persistency parameter $C$ measures the maximal number of transition between the local model $i$ and all other models in the time interval $(0, T), \gamma_{i}=\left[\gamma_{i}(1), \ldots, \gamma_{i}(T)\right] \in \mathbf{R}^{\mathrm{T}}, \dagger$ is the transposition operation, and $\|\cdot\|_{1}$ is the 1 -norm.

\section{b. $A I C$}

As was demonstrated in Horenko (2010c) in context of nonstationary inference, it is appropriate to use the AIC to determine the right-order parameters of the VARX model, i.e., the memory depth $m$, the number of cluster $K$, and the optimal BV persistency $C$. To select the proper order parameters [and the optimal functional for external factors $\phi\left(u_{t}\right)$ in Eq. (1)] for a given persistency parameter value [Eq. (18) of Horenko $(2010 \mathrm{c})^{2}$ ], the AIC is defined as

$$
\mathrm{AIC}=-2 \log L_{\max }+2 M
$$

where $L_{\max }$ is the maximum likelihood achievable by the model and $M$ is the number of free parameters. The lowest AIC is preferred. The resulting optimal Viterbi path provides a natural method for generating the climatology of a particular cluster state sequence. Cluster states are constructed by first assigning a model affiliation to each data point in the time series of anomalies according to the Viterbi path sequence $\Gamma(t)$. Then all anomalies for each given cluster state assignation are averaged. The averaged state is the composite or cluster state.

\section{c. Blocking indices}

We are interested in the utility of the FEM-BVVARX with regard to its ability to identify systematic changes in the regime states of the climate system. The dynamics of the midlatitude $\mathrm{SH}$ atmosphere are characterized by the presence of regimes represented by the southern annular mode (SAM) and high-low blocking

\footnotetext{
${ }^{2}$ For a complete description of the persistency parameter formulation, see section 2d of Horenko (2010c).
}

dipoles. To identify said regime states, the FEM-BVVARX must be able to accurately identify SH blocking. Meteorologists typically employ split-flow blocking indices to identify midlatitude blocking. Moreover such indices have been used to assess climate GCM performances (Tibaldi 1993) and to develop blocking climatologies (Pook and Gibson 1999). Pook and Gibson (1999) discuss in some detail the respective definitions of SH blocking and the development of the modern blocking index used at the Australian Bureau of Meteorology (BoM) as developed by Wright (1994) [see Shakina and Ivanova (2010) for a more general review of the literature on blocking indices]. This index is defined as

$$
0.5\left(U_{25}+U_{30}-U_{40}-2 U_{45}-U_{50}+U_{55}+U_{60}\right)>0,
$$

where $U_{y}$ represents the zonal component of the mean $500-\mathrm{hPa}$ wind (5-day averaged) at latitude $y$. The BoM index detects blocking whenever the index is positive. Large values indicate strong high and low latitude westerly winds or that the midlatitude westerly flow is weak. We will refer to Eq. (7) as the BoM index.

A related measure of SH blocking was developed by Tibaldi et al. (1994) in terms of mid- and high-latitude geopotential height gradients (GHGN and GHGS, respectively):

$$
\mathrm{GHGN}=\left[\frac{Z\left(\theta_{0}\right)-Z\left(\theta_{n}\right)}{\theta_{0}-\theta_{n}}\right]
$$

and

$$
\mathrm{GHGS}=\left[\frac{Z\left(\theta_{s}\right)-Z\left(\theta_{0}\right)}{\theta_{s}-\theta_{0}}\right],
$$

evaluated at each longitude point of the grid where $\theta_{n}=$ $35^{\circ} \mathrm{S}+\delta, \theta_{0}=50^{\circ} \mathrm{S}+\delta, \theta_{s}=65^{\circ} \mathrm{S}+\delta$, and $\delta=-5^{\circ}, 0^{\circ}$, $5^{\circ} .3 \mathrm{~A}$ given longitude is said to be blocked at time $t$ if, for at least one value of $\delta$,

$$
\text { GHGN }>0
$$

and

$$
\text { GHGS }<\frac{-10 \mathrm{~m}}{y^{\circ}}
$$

\footnotetext{
${ }^{3} \delta=2.5^{\circ}$ in Tibaldi et al. (1994), but often operationally as $5^{\circ}$ as used here.
} 

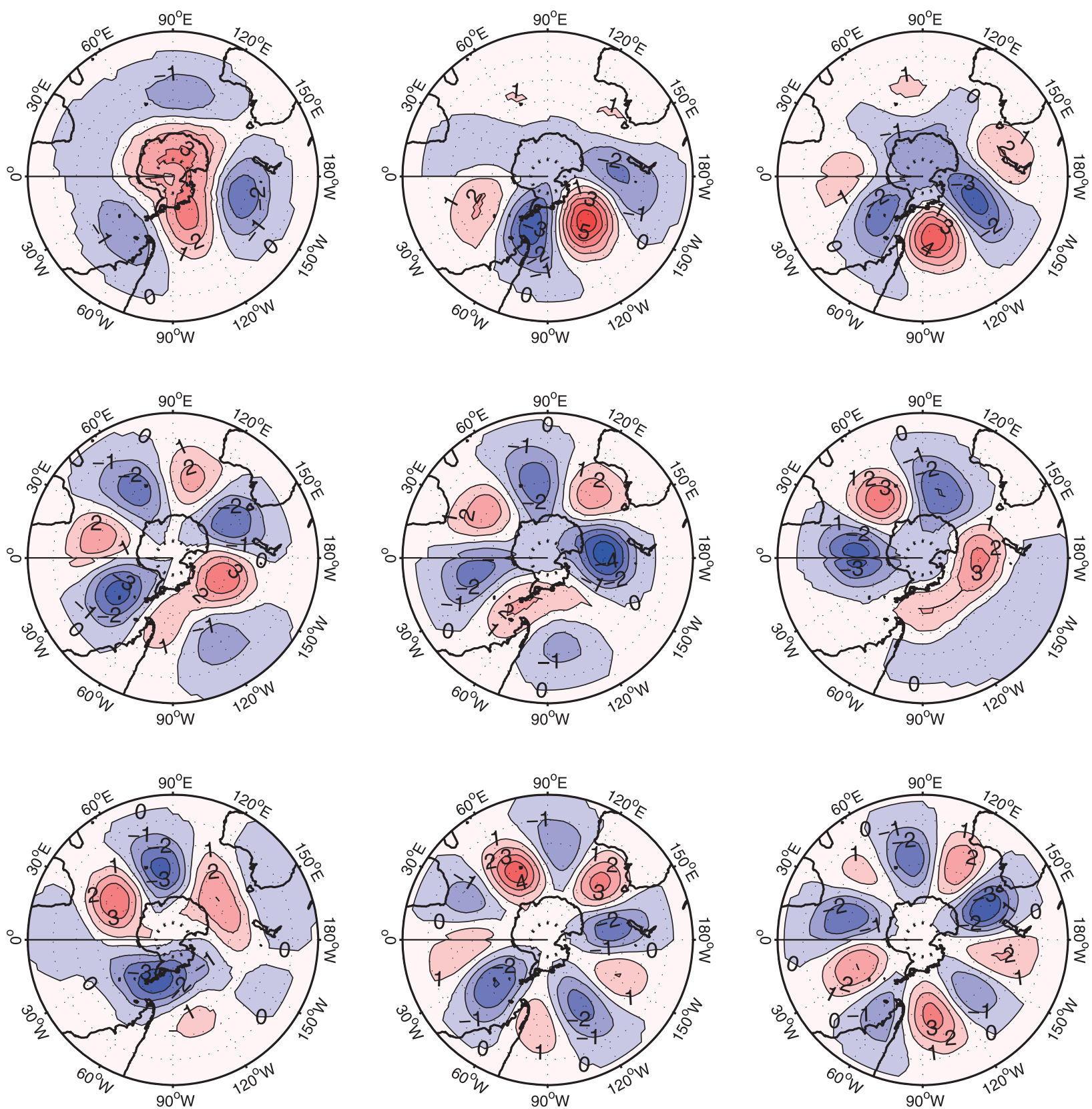

FIG. 1. (left to right), (top to bottom) Geographical distribution of first nine EOFs of the Southern Hemisphere 500-hPa geopotential height from NCEP-NCAR reanalysis data for the period January 1979-December 2009. The data cover the whole year, and a smooth annual cycle is subtracted: contour interval 1.

where $y^{\circ}$ is degrees latitude. Note that a 5-day running mean is typically first applied to the 500 -hPa data to isolate potential episodes of sufficient duration. The NOAA National Weather Service Climate Prediction Center defines a SH blocking index (strength) to be GHGN $\left[\mathrm{m}\left(y^{\circ}\right)^{-1}\right]$ [Eq. (8a)] minus mean observations at $500 \mathrm{hPa}$ (annual cycle), which we will refer to as the Tibaldi index. Equations (8) will be referred to as the Tibaldi blocking criterion. In the results that follow we examine both reanalysis and AMIP datasets comparing the BoM and Tibaldi indices to the FEM-BV-VARX analysis.

\section{Data}

In this study we compare and contrast results from reanalysis and model datasets. Primarily we examine the National Centers for Environmental Prediction 

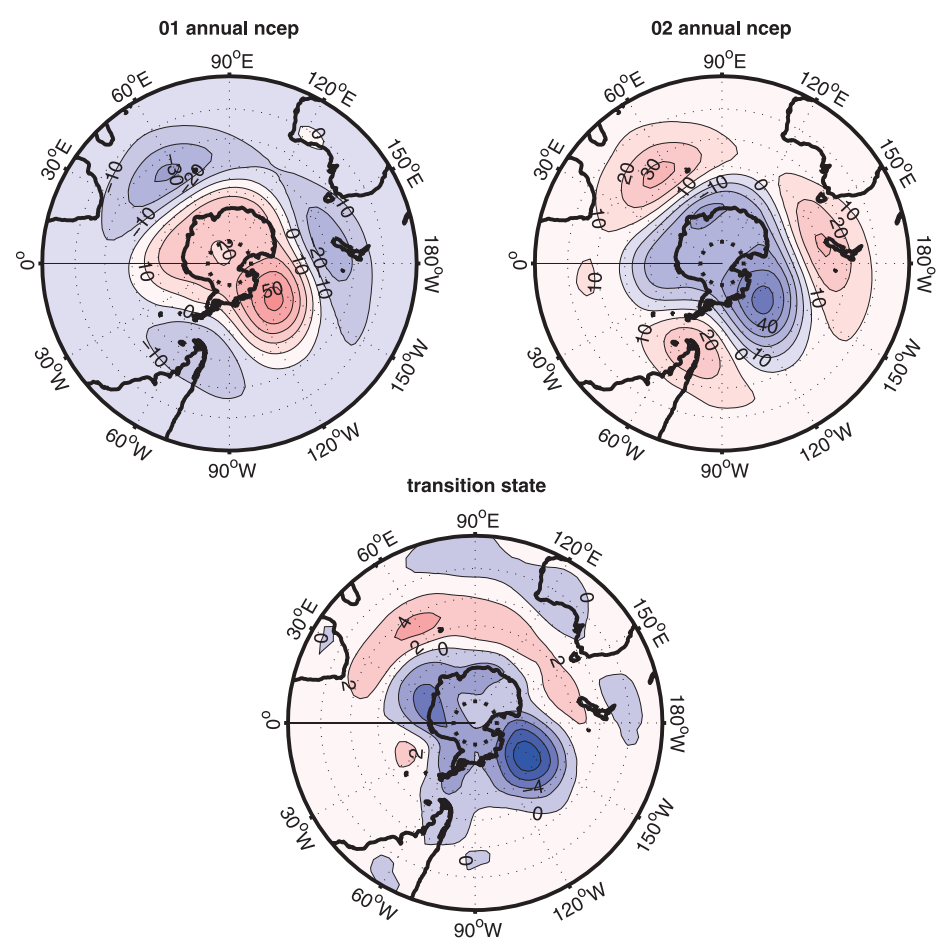

FIG. 2. Composites of 500-hPa geopotential height anomaly from NCEP reanalysis data covering the period 1948-2009 based on the FEM-BV-VARX Viterbi path and referred to as cluster (top left) state 1 and (top right) state 2 and (bottom) the transition state: averaged over all months of the year.

(NCEP)-NCAR reanalysis dataset using 500-hPa geopotential height fields in the Southern Hemisphere covering the period January 1948-December 2009. Owing to the dependence of $\mathrm{SH}$ observations on satellite data, reanalyses are not considered very reliable before about 1979 . However, we have chosen to include an analysis of the complete dataset, as we are considering only large-scale hemispheric features and
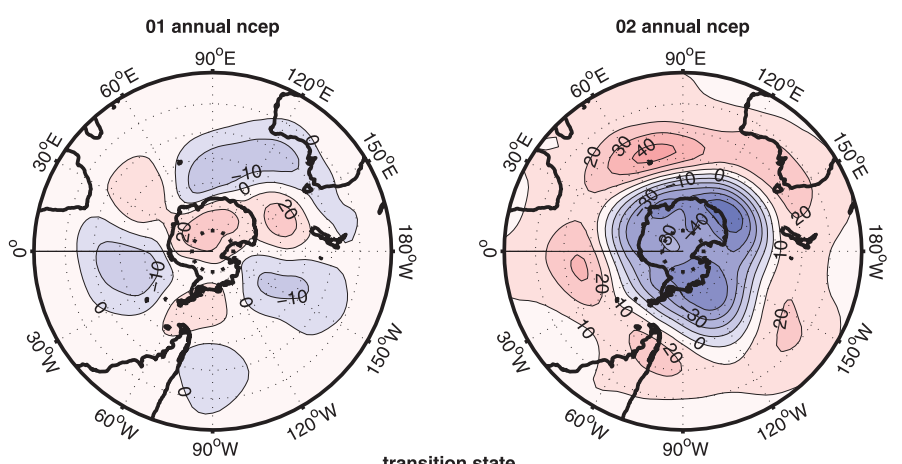

transition state

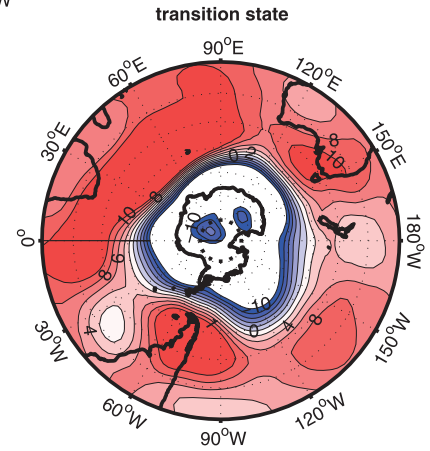

FIG. 3. As in Fig. 2, but for the period 1979-2009. 
consider reanalysis to be a reasonable "best guess." The FEM-BV-VARX algorithm requires continuous data, so we use data from the whole year. This approach is different to almost all other clustering studies, which have been focused on the NH winter season. We first subtract a smooth annual cycle from the data by computing the mean annual cycle by first taking the calendar average for each day. We also find that smoothing the noisy annual cycle via a running mean has little effect on the results.

We are also eager to consider the utility of the clustering approach as a primary model evaluation tool. To this end, we consider model data in the form of a 30-yr model run from 1979 to 2008 using the Atmospheric Model Intercomparison Project (AMIP) protocol (Gates 1992). The AMIP run employs observed monthly varying sea surface temperature (SST) and sea ice distributions. Concentrations of greenhouse gases are fixed in the model and ozone concentrations are set to climatological monthly zonal mean values. As the concentrations of these gases have changed over the period of the run, impacting drivers such as the SAM (Polvani et al. 2011) and blocking, the model run provides a baseline for comparison to the reanalysis. The model used for the simulation described here is a version of the atmospheric general circulation model (AGCM) within the Australian Community Climate and Earth System Simulator (ACCESS). The AGCM is the U.K. Met Office Unified Model (UM) (Martin et al. 2006). The model code used in this experiment is the Met Office UM6.6 and the climate configuration used is that of the Hadley Centre Global Environmental Model, version 2 (HadGEM2), version r1.0 (Collins et al. 2008; Rashid et al. 2009). The model has a horizontal resolution of N96, equivalent to a $1.25^{\circ}$ latitude $\times 1.875^{\circ}$ longitude grid, and 38 vertical levels.

\section{EOF analysis}

To reduce the dimensionality of the reanalysis and model data prior to applying the FEM-BV-VARX method, we utilize standard EOF analysis. For the calculation of the covariance matrix, the field is properly area weighted by the cosine of the latitude. We use the $L_{2}$ norm. The first EOF explains about $10 \%$ with a smooth decay of the explained variance for the following EOFs. The first 10 EOFs cumulatively explain 54\%, the first 20 EOFs cumulatively explain $75 \%$, and the first 60 EOFs cumulatively explain $95 \%$ of the total variance. The first 130 are needed to explain $99 \%$ of the total variance.

The geographical distributions of the first nine EOFs are displayed in Fig. 1. The first EOF represents the wellknown southern annular mode. EOFs 2-9 represent synoptic-scale wave trains while EOFs $10-17$ contain more small-scale structures (not shown). As midlatitude blocking in the $\mathrm{SH}$ is predominantly a wavenumber-3

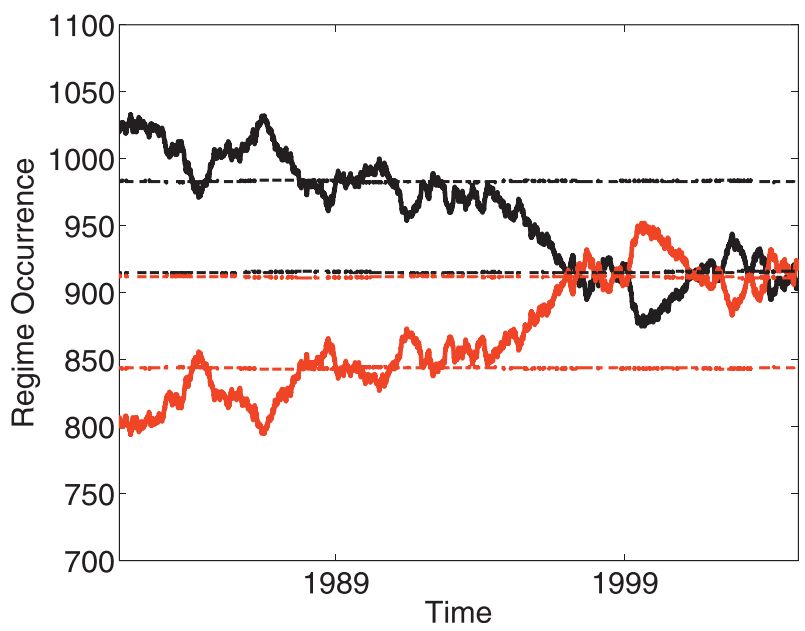

FIG. 4. Five-year running mean of regime occurrence frequency from FEM-BV-VARX calculation: blocking pattern (black) and the southern annular mode (red). A Markov chain is fitted to the hidden state sequence, which acts as a model of background variability. The dashed lines denote the 10th and 90th percentiles of a 1000-member ensemble generated from the corresponding Markov transition matrix.

process, we determine that reducing the dimension of the data to the first nine EOFs should be sufficient. However, for completeness, the role of the higher order EOFs is quantified through sensitivity studies of the clustering algorithm using 9 and 20 EOFs, respectively. It is found that, while the basic structure of the composite cluster states is relatively insensitive to the higher-order EOFs, the relative amplitude of the cluster state structures to some degree are. The cluster algorithm iterates over the principal components calculated from the EOFs and anomaly datasets. The identified trends in transitionfrequency, residence times, and persistency identified in the datasets are insensitive to whether 9 or 20 principal components are used in the dimension reduction.

\section{Results and discussion}

In this section, we consider cluster states, transition frequency, time of residency, and regime occurrence frequency in the NCEP reanalysis over the periods January 1948-December 2009 and January 1979December 2009. We will compare Viterbi paths from the FEM-BV-VARX analysis to the representation of blocking derived from the BoM and Tibaldi indices and contrast composite states for the Southern Hemisphere and the Australian blocking region. A sensitivity analysis of the FEM-BV-VARX method to number of EOFs, domain, and smoothing parameter was first conducted to provide a baseline for the subsequent analysis. As noted, earlier results are very similar whether 9 or 20 EOFs were used to reduce the dimensionality of 

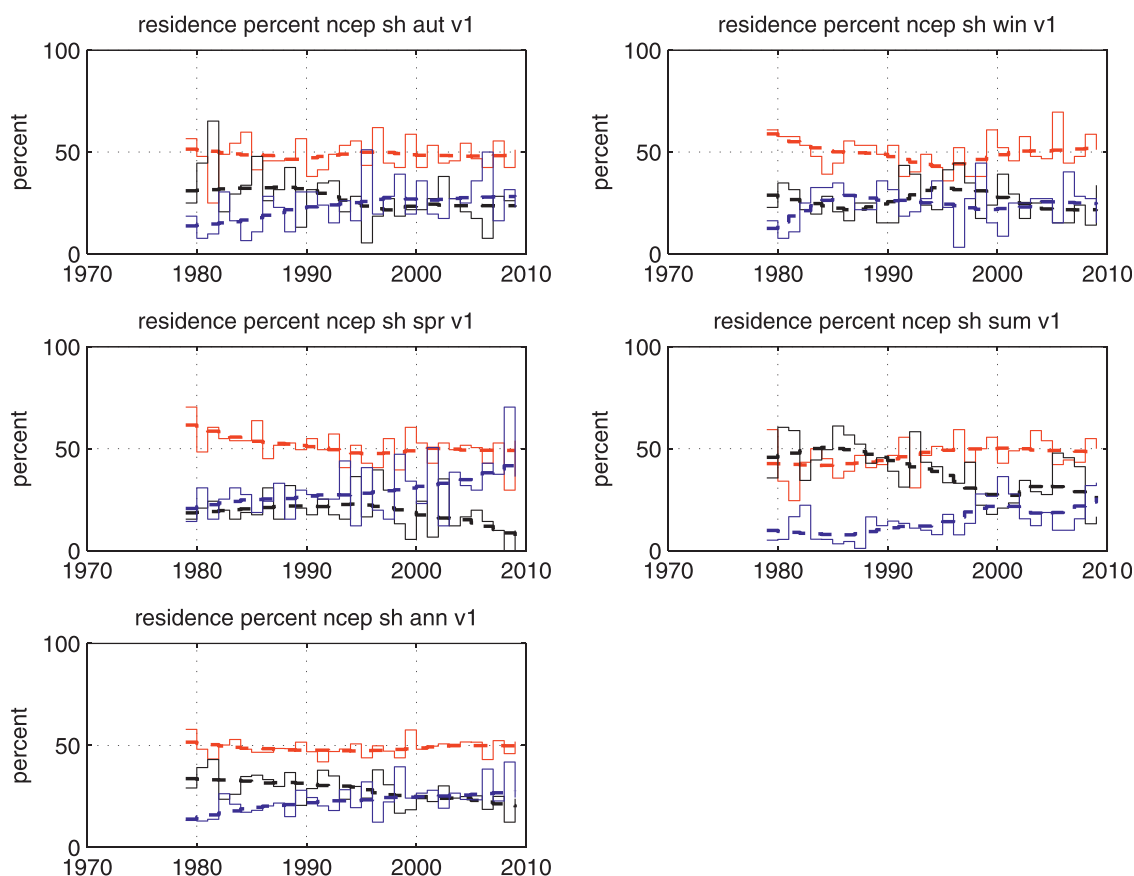

FIG. 5. Percentage of resident time in blocked (black), SAM (blue), or transition states (red) for NCEP 500-hPa reanalysis 1979-2009 5-day running average for each of the seasons autumn (aut), winter (win), spring (spr), summer (sum), and the annual (ann) period. The dashed lines are a LOESS fit to the time-averaged data. The solid lines are the values and averaging periods of the data.

the data. Further, the optimal memory depth [lag $m$ in VARX model of Eq. (1)] determined by lowest Akaike value is uniformly $4-5$ days, consistent with blocking predictability.

\section{a. NCEP reanalysis}

The FEM-BV-VARX analysis of the NCEP reanalysis over the period 1948-2009 reveals two significant metastable states (Fig. 2). The geographical structure of these two states are computed by using the model affiliation or Viterbi path sequence (or equivalently hidden state sequence), while the number of significant hidden states has been estimated by use of the AIC criterion, Eq. (6). According to the hidden state sequence, conditional composites are computed based on the full $500-\mathrm{hPa}$ geopotential height field (annual cycle subtracted). Both hidden states have a wavenumber-3-like structure with positive/negative anomalies over South America, south of Tasmania, and southwest of South Africa. Neither of the hidden states project strongly on any of the leading 20 EOFs used in the clustering analysis; however, the anomaly structures are located in each of the three SH blocking regions. As such, we will refer to the top left (top right) panel of Fig. 2 as the negative (positive) SH blocking pattern(s). Throughout this section the persistency parameter is set to zero. Analogous to the transition state used by Tibaldi et al. (1994), we also define a transition state according to the following: an anomaly at any particular time will be assigned to the transition state if for any given 5-day period the model affiliation sequence (Viterbi path) occupies no more than 3 days in any given state. A composite of the transition state is then constructed by averaging all anomalies assigned to be in the transition state (Fig. 2, bottom). We refer to FEM-BV-VARX where the persistency parameter has been set to zero as being "nonsmoothed."

In Fig. 3 the clustering analysis over the period 19792009 is shown. Figure 3 (left) closely resembles the positive SH blocking pattern; however, hidden state 2 (Fig. 2, top right) has a strong resemblance to the positive phase of the southern annular mode. Hidden state 2 has a strong annular structure and projects strongly onto EOF1 (Fig. 1), whereas the transition state for the period 1948-2009 (Fig. 2, bottom) is weak and unstructured; apart from some projection onto the $150^{\circ} \mathrm{W}$ negative anomaly between New Zealand and Chile, the transition state for the period 1979-2009 (Fig. 3, bottom) is clearly SAM-like. ${ }^{4}$

\footnotetext{
${ }^{4}$ While the concept of a transition matrix has some utility here, in later AMIP cases where $C \neq 0$ (smoothed) we will show that a transition matrix is redundant.
} 

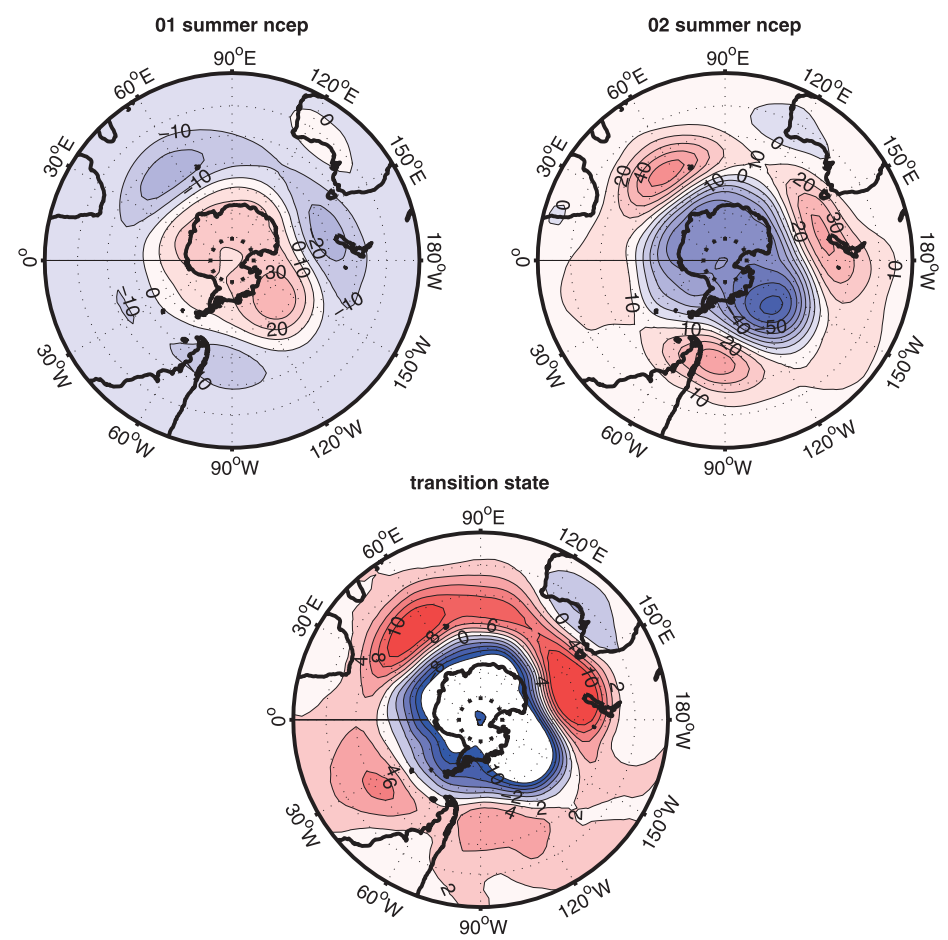

FIG. 6. NCEP 500-hPa cluster (top left) negative and (top right) positive blocking and (bottom) transition states 1948-2009 summer averaged.

\section{1) INTERANNUAL VARIABILITY}

To investigate the interannual variability of the hidden state occurrences and to connect to the earlier $\mathrm{NH}$ study of Franzke et al. (2009), we compute a 5-yr running mean of the hidden state sequence (Fig. 4) of the FEM-BV-VARX. We also fit a Markov chain to the hidden state sequence, which acts as a model of
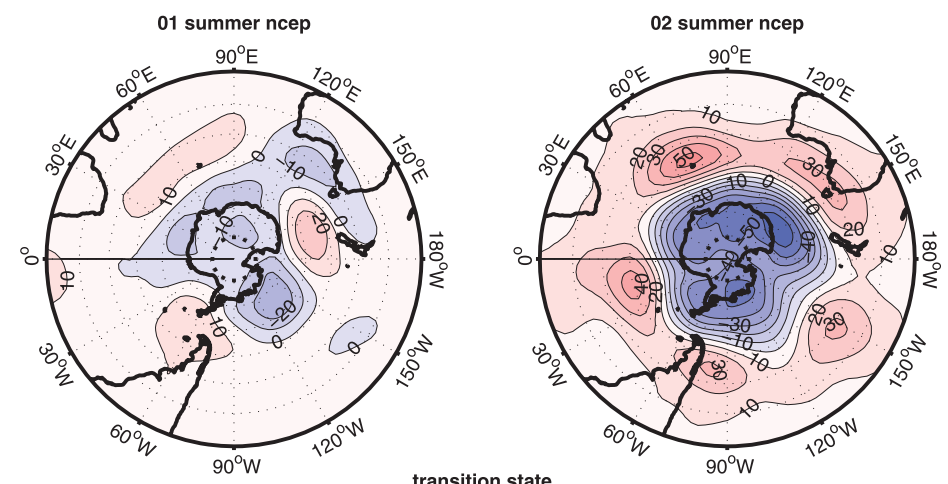

transition state

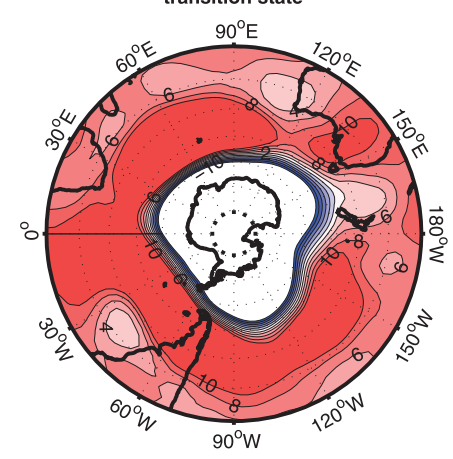

FIG. 7. NCEP 500-hPa cluster (top left) positive blocking and (top right) zonal and (bottom) transition states for the period 1979-2009. 


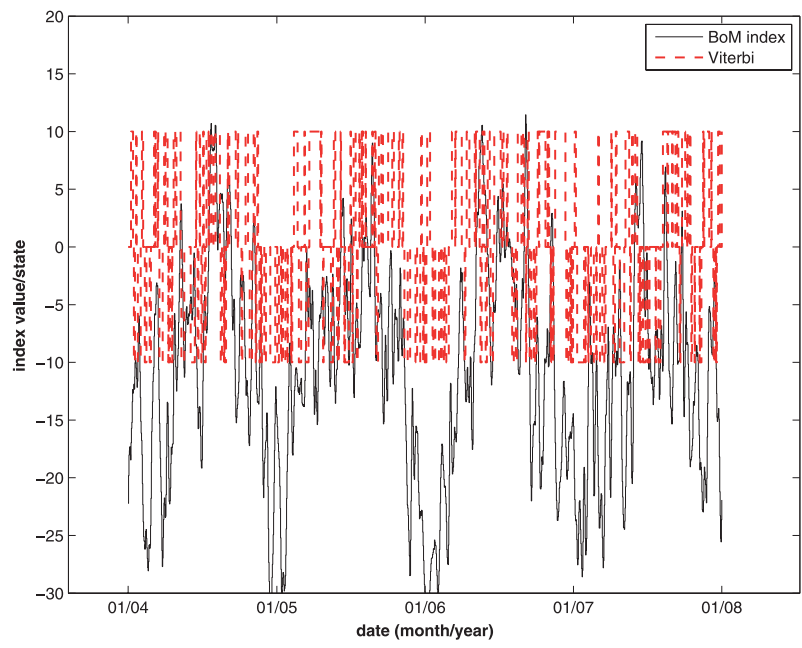

FIG. 8. Comparison of FEM-BV-VARX Viterbi paths (nonsmoothed) to the BoM and Tibaldi blocking indices (5-day running mean) for NCEP $500 \mathrm{hPa}$ over the period January 2004January 2008. Note that FEM-BV-VARX Viterbi paths have been scaled for comparison here such that +10 is the positive blocking state, 0 is the transition state, and -10 is the negative blocking state.

background variability. This calculation highlights that the observed interannual variability is unlikely due to intrinsic variability or non-Markovian processes. As can be seen, the hidden state frequency of occurrences exhibit strong trends. Hidden state 1 (hemispheric blocking), which was dominating in the early 1980s, shows a gradual decrease in its frequency of occurrence, while hidden state 2 (SAM) shows a corresponding increase in its frequency of occurrence. These results also give the impression that the trend is leveling off in the 2000s. This interpretation is consistent with the nonlinear trend analysis of the SAM in Franzke (2009), which also reveals that the trend in the SAM is leveling off in the late 1990s and early 2000s. Overlaid on these long-term trends are other fluctuations, which are likely genuine internal variability. These fluctuations are of a magnitude one can expect from sampling variability of a Markov chain. Conditional means (calculated using the first nine EOFs) of the hidden states for the period 1979-83 and 2005-09 (not shown) suggest that hidden state 1 corresponds to a blocking-like state over the southern tip of South America in the early period. In the latter period, there is strengthened blocking south of Australia and a strengthened jet stream east of Victoria Land. In comparison to the $1979-83$ period the latter period hidden state 2 is much more zonally symmetric and strongly resembles the positive phase of the SAM.

To look at the seasonal trends, we calculated the annual and seasonal mean regime occupancies (using the FEM-BV-VARX method). These calculations (not shown) reveal that the largest trend occurs in summer [December-February (DJF)] and the spring post-2000 [September-November (SON)] with no trend during winter [June-August (JJA)] and autumn (SON). Substantial interannual variability was also observed. In Fig. 5 we consider the percentage of time resident in a given state each year for the 1979-2009 period after taking a 5-day running average. There is no visible trend for the transition state in any season despite the significant residence percentage $(\sim 50 \%)$. As with the 5-yr running means, clear trends were observed in summer and spring. Summer-averaged composite states calculated over the 1948-2009 period reveal a strong positive SH blocking pattern (Fig. 6, top right) and a weak negative blocking state (Fig. 6, top left), whereas the transition state (Fig. 6, bottom) has significant amplitude and is unmistakably SAM. The utility of the transition state is now clear. In
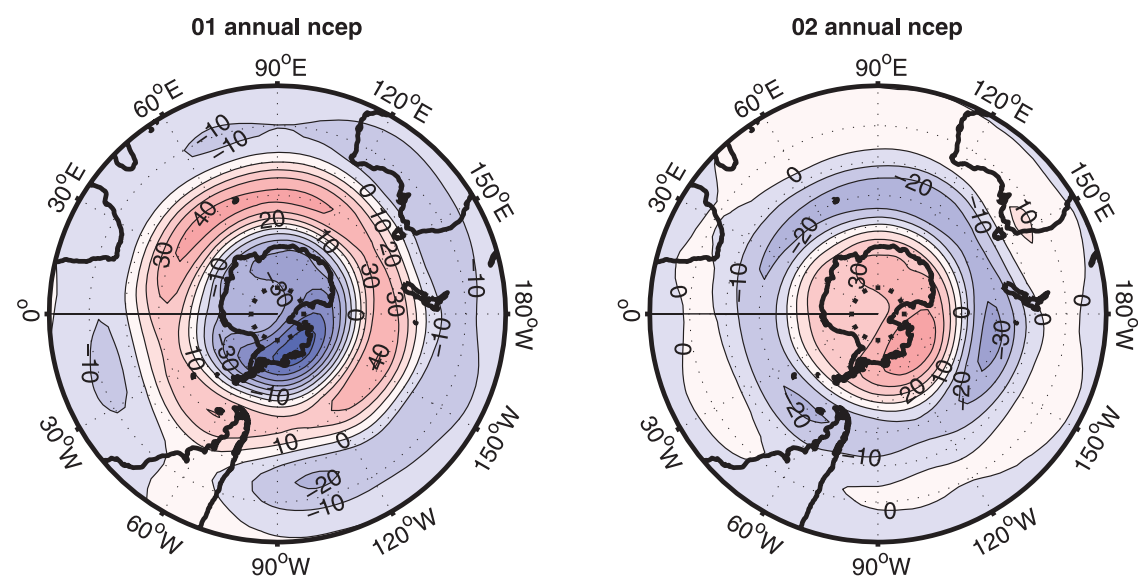

FIG. 9. Composite states using the BoM index for NCEP 500 hPa 1948-2010: Southern Hemisphere averaged over all seasons. 

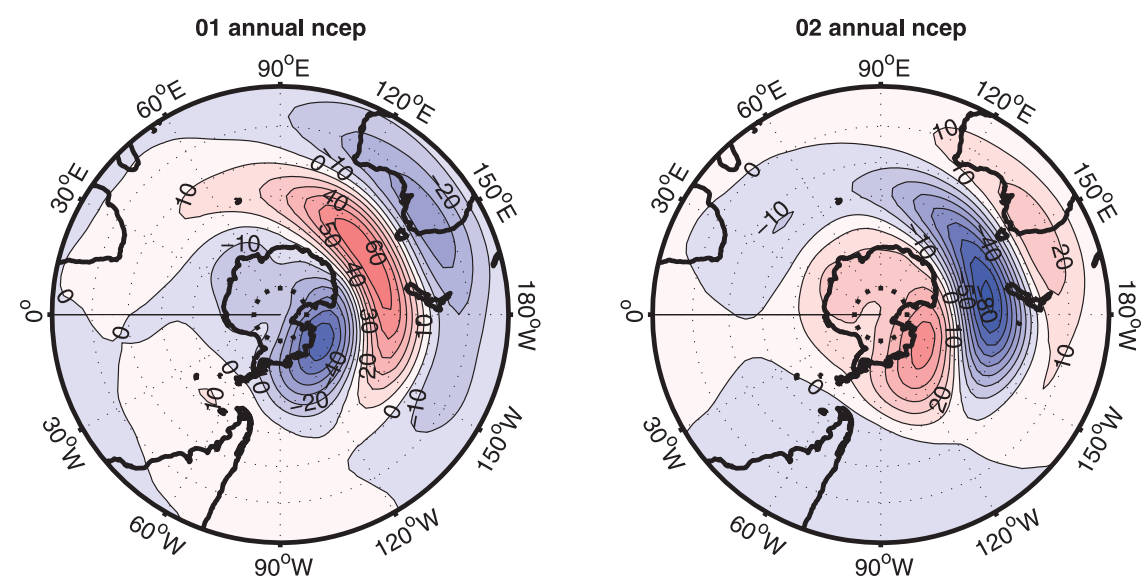

FIG. 10. As in Fig. 9, but for the Australian region (longitude sector $110^{\circ}-210^{\circ} \mathrm{E}$ ) averaged over all seasons.

Fig. 6 we see that, over the summer periods during 1948 2009, the negative SH blocking regime state weakens and SAM begins to replace it as a true metastable state. The increasing dominance of SAM is more clearly observed during the 1979-2009 summer periods with an almost totally degraded negative SH blocking pattern replaced by a very strongly emerging SAM (Fig. 7, top right) and in the transition state (Fig. 7, bottom). The positive blocking phase remains robust but weakened (Fig. 7, top left).

\section{2) COMPARISON OF BLOCKING INDICES}

As noted in section 3a the FEM-BV-VARX analysis is relatively insensitive to dimension reduction as long as nine or more principal components are used. Throughout this analysis, we set the persistency parameter to zero in the FEM-BV-VARX. Figure 8 compares the BoM blocking index to the FEM-BV-VARX Viterbi paths over the period January 2004-January 2008. This period was arbitrarily chosen, but is a representative snapshot. The Viterbi paths are either in the positive $(+1)$ SH blocking state, negative $(-1)$ blocking state, or SAM and the transition state (0). The BoM index is considered to be in a blocking state whenever positive. We first note the qualitative agreement between the BoM index and FEM-BV-VARX Viterbi paths over this period with both indices reflecting the seasonal cycle in blocking activity.

We now calculate composites from the BoM blocking index by considering all instances where the BoM index is greater than one standard deviation above the mean to be in the $(+1)$ state and all instances where the BoM index is less than one standard deviation below the mean to be in the $(-1)$ state. This is carried out for both the Southern Hemisphere (Fig. 9) and the Australian blocking region, defined here as the longitude sector $110^{\circ}-210^{\circ} \mathrm{E}$ (Fig. 10).
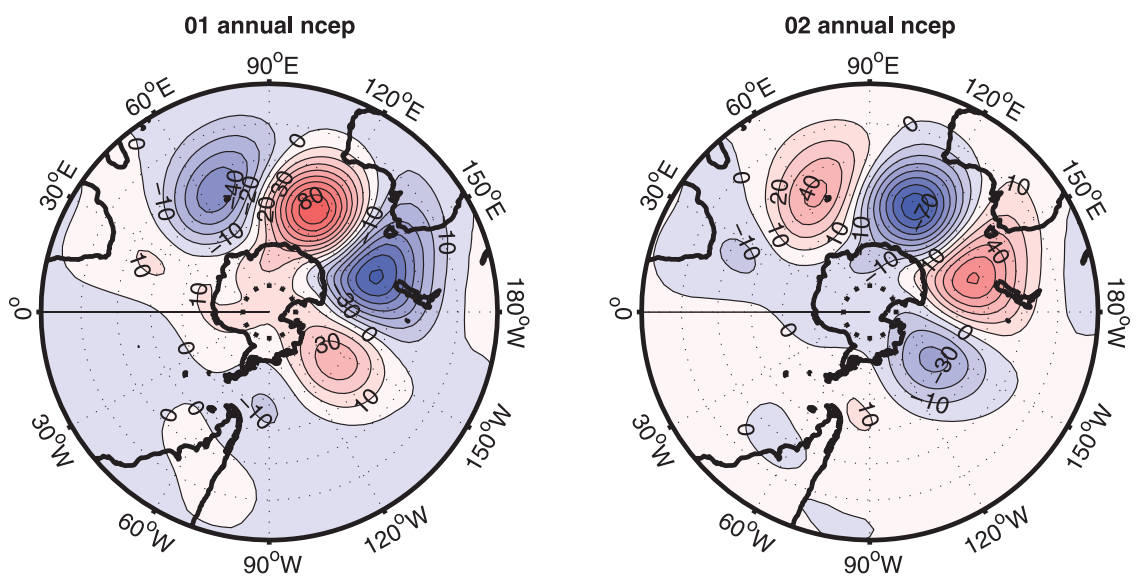

FIG. 11. As in Fig. 9, but using the FEM-BV-VARX Viterbi paths: Australian region (longitude sector $110^{\circ}-210^{\circ} \mathrm{E}$ ) averaged over all seasons. 

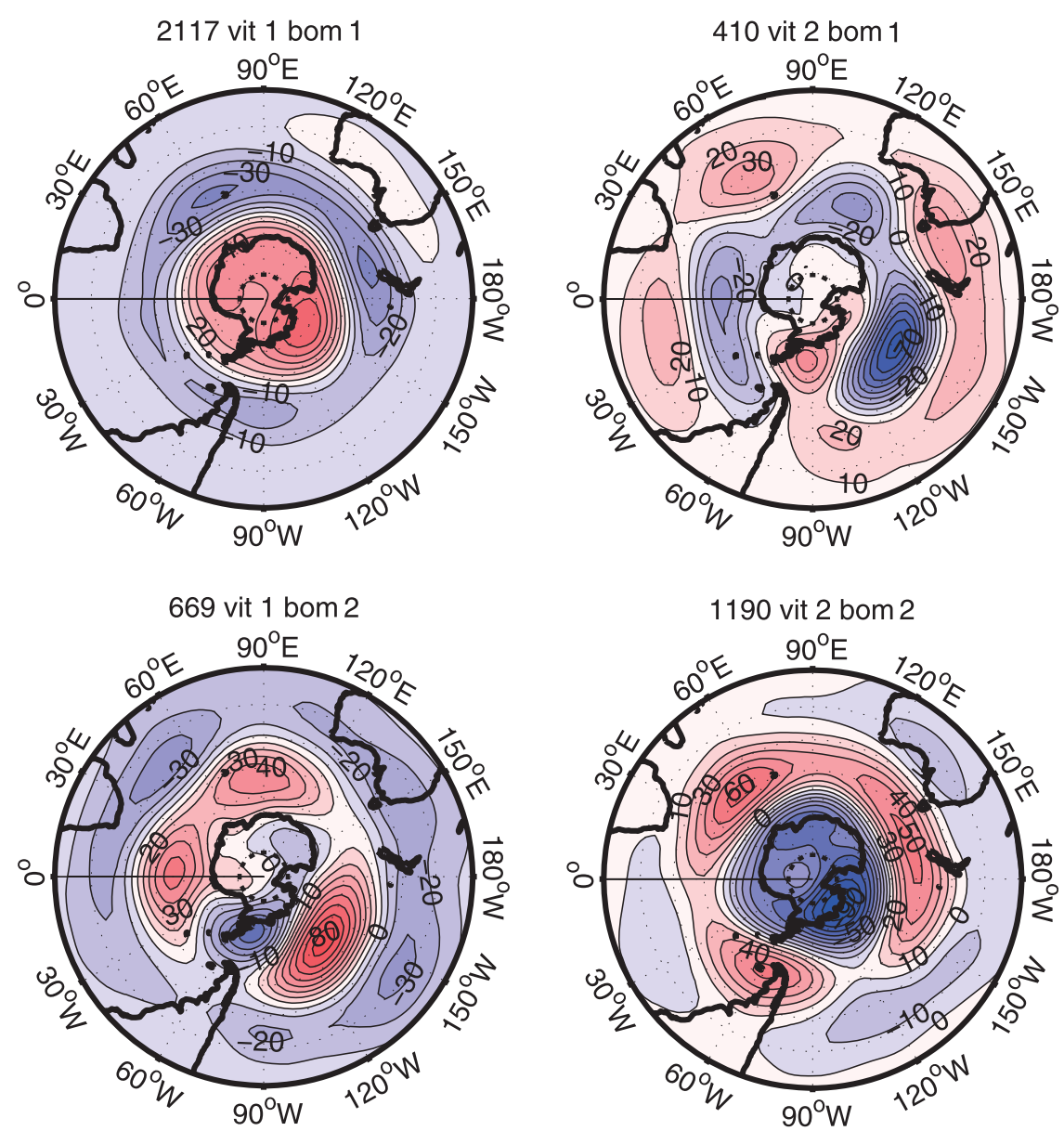

FIG. 12. Composite states for NCEP 500 hPa 1948-2009: cases where (top left) BoM (bom) and FEM-BV-VARX (vit) are both in the negative blocking state (as in state 1, Fig. 2) and (top right) FEM-BV-VARX is in positive blocking state 2, BoM is in negative blocking state 1 . (bottom left) FEM-BV-VARX is in state 1, BoM is in state 2. (bottom right) Both FEM-BVVARX and BoM are in the positive blocking state (as in state 2, Fig. 2). The numbers above each plot refer to the number of days in each composite case.

Despite the clear similarities between the global BoM index and the Viterbi paths in Fig. 8, there are manifest differences between the BoM and FEM-BV-VARX composite states. Ideally blocking composites from any method should resemble the "canonical" block. For the Australian region this would typically consist of anticyclones in the latitude band $35^{\circ}-60^{\circ} \mathrm{S}$ most commonly in the Tasman Sea but also occurring in the western Pacific, Great Australian Bight, and eastern Indian Ocean (Baines 1983; Trenberth and Mo 1985). While splitting of the basic westerly flow implies a high-low dipole, blocking in the Australian region is typified by a larger and more conspicuous high (Wright 1974) or sequence of highs (Baines 1983).

As the FEM-BV-VARX method is not descriptive and makes no assumptions about the structure of blocks, there is no a priori guarantee that the cluster composites will resemble structures that look like blocks. However for the Australian region the FEM-BV-VARX produces composites with coherent structures that are confined to the midlatitude jet with maxima in the locality of the Tasman Sea (Fig. 11). More generally the global FEMBV-VARX composites (Figs. 2 and 3) show structures immediately recognizable as coherent blocked states and do, in fact, match fairly precisely the features of blocking in the Southern Hemisphere (Noar 1983; Trenberth and Mo 1985; Jones and Simmonds 1994; Sinclair 1996). That is, the global FEM-BV-VARX composites show metastable states with the classic three-wave blocking structure in the Southern Hemisphere with nodes in the dominant blocking regions, consistent with theory (Frederiksen 1982, 1983; Zidikheri et al. 2007; O'Kane and Frederiksen 2008), observational (Trenberth and Mo 1985; Jones and 


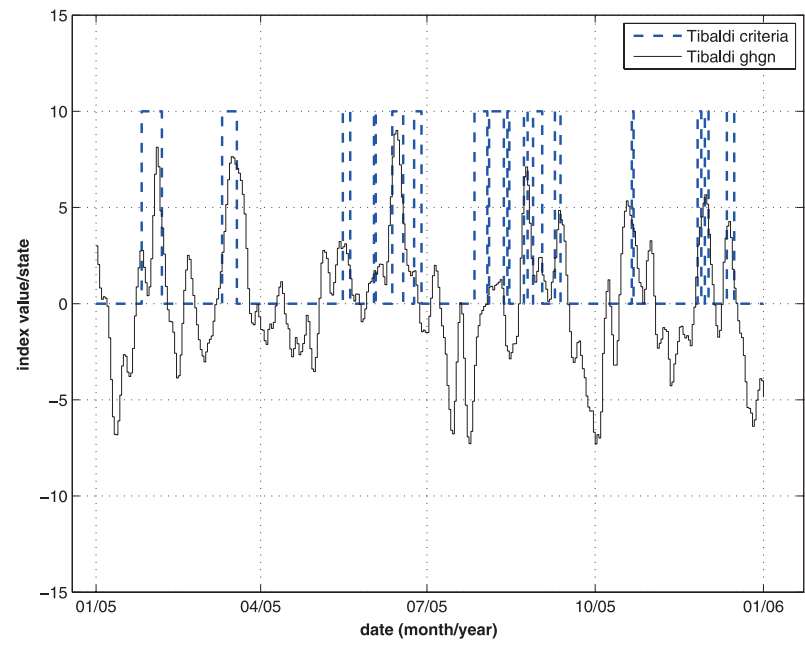

FIG. 13. Comparison of Tibaldi index (GHGN) vs criteria for the period January 2005-January 2006 over the Australian region. Note that the annual cycle has been removed from the Tibaldi index.

Simmonds 1994; Sinclair 1996), and numerical weather prediction (Noar 1983) studies.

By contrast, for the Australian region the BoM splitflow index generates blocking composite states with anomalies across a large latitudinal band that straddles both subtropical and polar jets, consistent with a splitting of the flow (Fig. 10). For the global BoM index composites (Fig. 9) the three-wave structure is weak and the nodes are displaced from the regions usually associated with persistent height anomalies in the literature (Jones and Simmonds 1994; Sinclair 1996) and observed in individual case studies (Noar 1983; Trenberth and Mo 1985). Near-identical structures (not shown) to Figs. 9 and 10 were found using the Tibaldi index, Eq. (8a) of section 2 c.

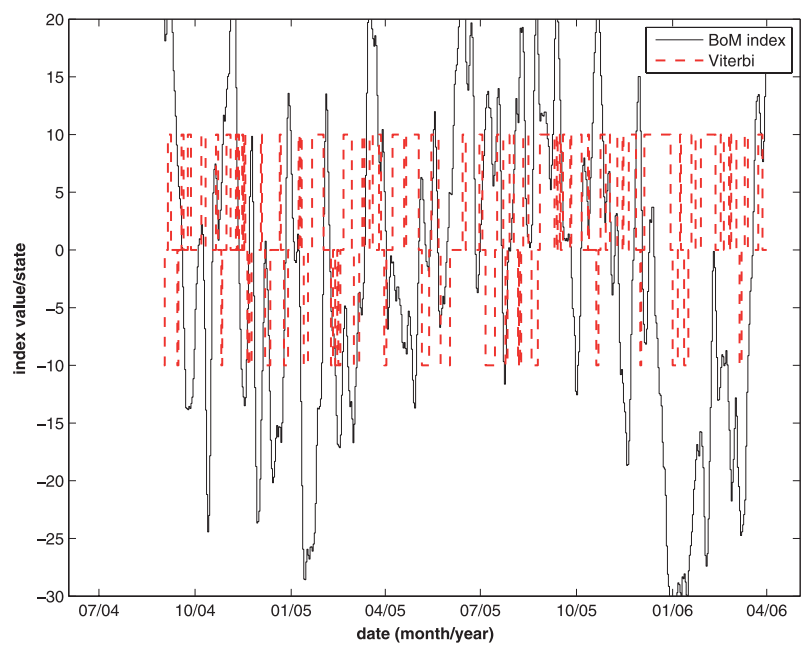

FIG. 14. Comparison of FEM-BV-VARX Viterbi path vs BoM index for the period September 2004-April 2006 over the Australian region.

In Fig. 12 we further consider the relative correspondence between the BoM index and the FEM-BV-VARX composites for the entire hemisphere over 1948-2009. Here we generate composite height anomalies for the combinations of days in which the FEM-BV-VARX and BoM indices do and do not indicate blocking. The topleft plot in Fig. 12 shows composites for cases in which both indices are in the negative blocking state and the bottom-right plot shows the composite where both indices are in the positive blocking state. These composites show the three-wave blocking structure characteristic of the FEM-BV-VARX metastable states (Fig. 2). The indices are in agreement on the blocking and negative blocking state for about two-thirds of the total number of days compared. The top-right and bottom-left
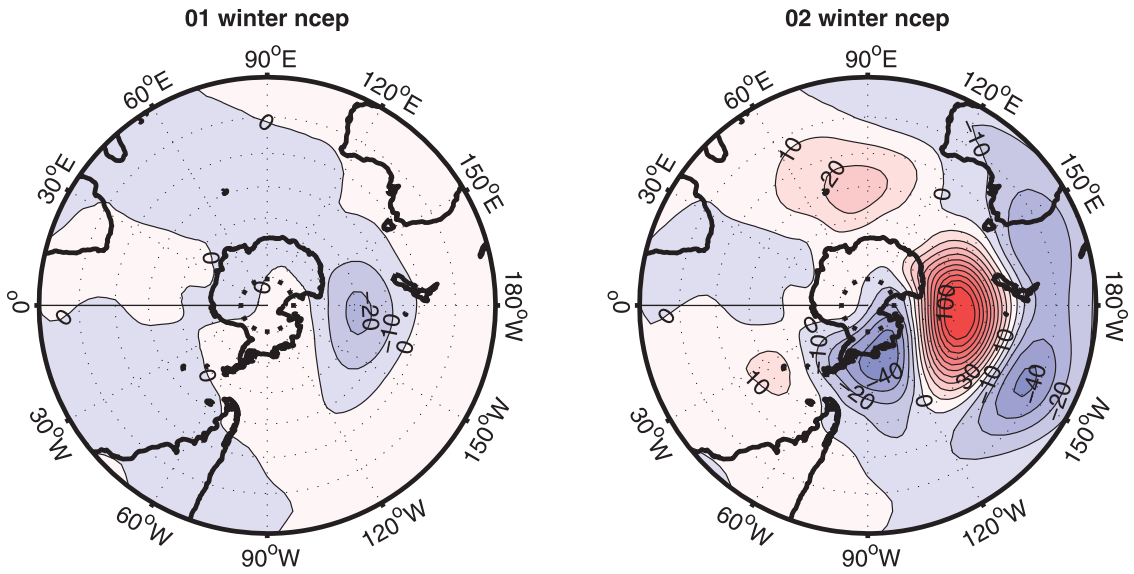

FIG. 15. Anomaly composites for the Tibaldi criteria for the period 1948-2009 for all winters over the Australian region corresponding to (left) all nonblocked times and (right) all blocked states. 


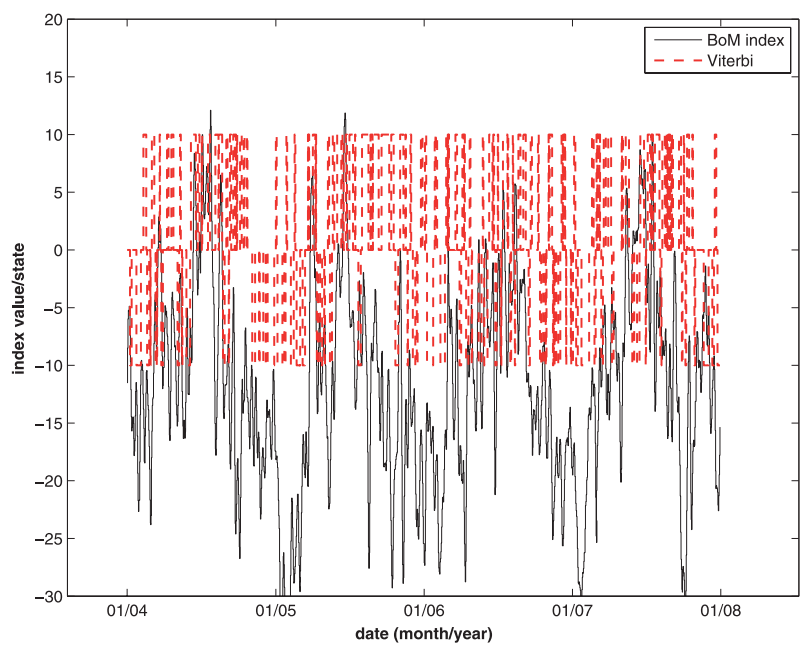

FIG. 16. Comparison of VAR-FEM Viterbi paths (no smoothing) to the BoM blocking index (5-day running mean) for AMIP $500 \mathrm{hPa}$ : the period January 2004-January 2008 is depicted.

plots correspond to composites over days in which the two blocking indices are not in agreement because one index is in the positive blocking state and one index is in the negative blocking state. These cases account for about a one-third of the total number of days.

Comparison of the Tibaldi index and criteria in Fig. 13 contrasts blocking strength against blocking state as defined by Eq. (8a) and Eqs. (8), respectively, demonstrating that the Tibaldi index is an incomplete approximation to the full blocking criterion. Similarly, Fig. 14 compares FEM-BV-VARX Viterbi paths and BoM index over the Australian region with clearly evident differences. Comparison of the relative frequency of blocking as defined by FEM-BV-VARX Viterbi paths and the Tibaldi criteria shows much reduced blocking occurrence in the Tibaldi criteria. The Tibaldi index is observed to be largely in phase with the BoM index.

In Fig. 15, we show anomaly composites using the Tibaldi criteria for the Australian region for all winters over the period 1948-2009. The composites are for both blocked and nonblocked states. The blocked state (Fig. 15, right) is a very close match to Fig. 21a of Tibaldi et al. (1994) and closely resembles observed trends in geopotential height toward the high index polarity of the SAM as reported in Fig. $3 b$ of Thompson and Solomon (2002). While the mean nonblocked state (not shown) is zonal, the composite of perturbations about the average nonblocked zonal flow (Fig. 15, left) is largely unstructured. Tibaldi et al. (1994) assumed that the nonblocked mean state, averaged over 7 years of weather forecast data, is zonal (not shown), which is in general agreement with the results of our analysis of the NCEP reanalysis.

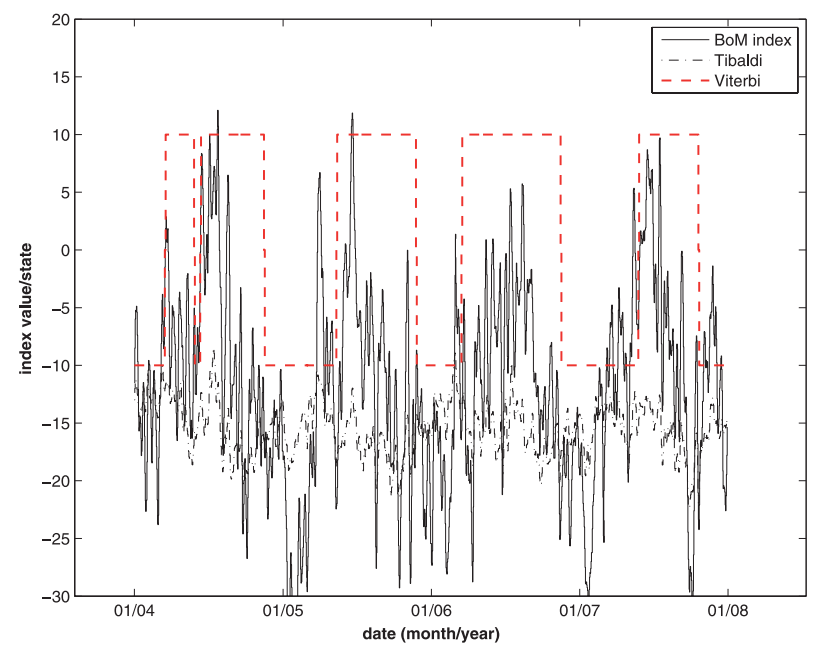

FIG. 17. As in Fig. 16, but with the Tibaldi index included and a nonzero smoothing (persistency parameter set to 100) set in the FEM-BV-VARX. This figure clearly displays the tendency of the AMIP simulation to lock into one state for long periods.

\section{b. AMIP model data}

In this section we consider the AMIP model run described in section 3 over the period January 1979December 2008. As noted the AMIP run uses prescribed sea surface temperature, sea ice concentrations, climatological greenhouse gas (GHG), and ozone concentrations. The interest here is to contrast the results from the NCEP dataset with the AMIP run in order to indicate differences in the behavior of the SAM and blocking to GHG and ozone forcing. We will also consider cases in which the persistency parameter is nonzero to ascertain the impact of smoothing on the results.

\section{COMPARISON OF BLOCKING INDICES}

In Figs. 16 and 17 we compare BoM and Tibaldi indices and FEM-BV-VARX Viterbi paths over a 4-yr subset of the model run. Figure 16 has the persistency set to zero, while Fig. 17 has a persistency parameter value of 100 that was determined to be the optimal parameter setting with the lowest AIC. ${ }^{5}$ We again note the close correspondence between BoM and Viterbi paths throughout the entire period. BoM and Tibaldi indices are seemingly in phase, although the Tibaldi index always remains in the same state. The role of the persistency parameter as a smoother (Fig. 17) is now obvious, demonstrating that the AMIP model has a strong seasonal cycle locking into a positive blocking state $1(+10)$ in

\footnotetext{
${ }^{5}$ The results using NCEP data were qualitatively unchanged by smoothing.
} 

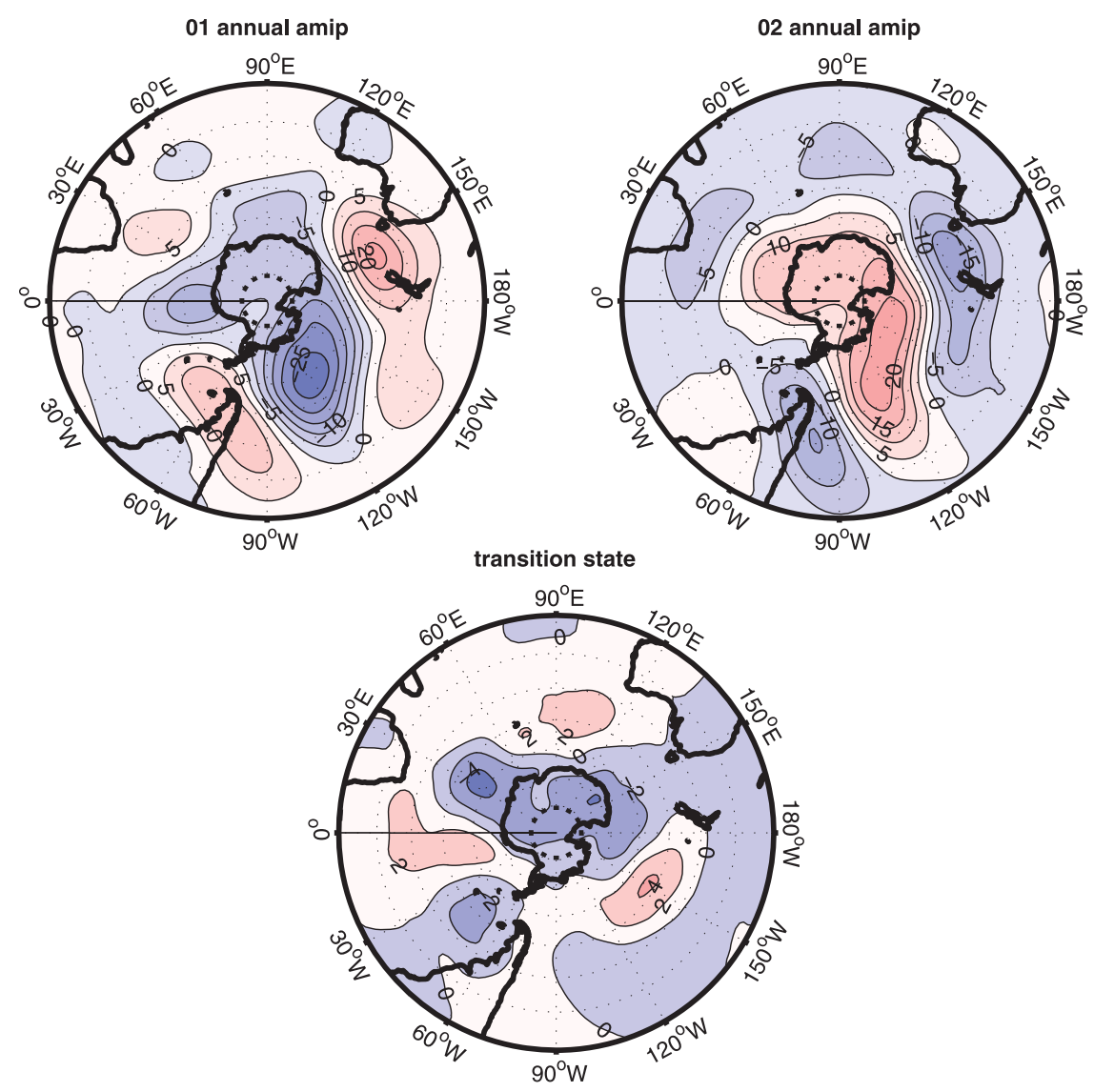

FIG. 18. Composites using the Viterbi paths, shown in Fig. 16, for AMIP 500 hPa 1979-2008: persistency parameter set to 0 .

winter (JJA) and spring (SON) and a negative blocking state $2(-10)$ during summer (DJF).

The metastable states in the AMIP run correspond to positive and negative phases of blocking (Fig. 18). Calculating a transition state (Fig. 18, bottom) from the FEM-BV-VARX Viterbi paths in Fig. 16 reveals almost no structure despite a residence percentage time of about $50 \%$ (Fig. 19). An examination of the seasonally averaged residence time (Fig. 19) shows significant trends in autumn and winter with little or no trend in summer and spring such that the annual trend is entirely due to an increased autumn-winter residence time. The transition state exhibits no trend, as expected. Results from the smoothed FEM-BV-VARX (Figs. 20 and 21) are entirely consistent with the unsmoothed case with positive/ negative hemispheric blocking states (Fig. 20). The residence periods (lengths) are shown in Fig. 21, where we clearly see an increasing preference to remain in state 1 over the last decade of the model run for winter and a similar but weaker trend in autumn. The model system locks almost exclusively into the positive blocking state in spring and into the negative blocking state in summer.

\section{Summary and conclusions}

We have applied a nonstationary clustering method (FEM-BV-VARX) to calculate the metastable states of the SH over the NCEP reanalysis period. We find that post-1978 there has been a significant decline in blocking over the summer months and during spring. Post-2000, however, there is little evidence of trends in autumn and winter. Similar trends, consistent with the satellite period, are also observed over the full reanalysis period and, while most significant in summer and spring, are also observable in winter and autumn. For the satellite period, the FEM-BV-VARX metastable states are clearly SAM and a hemispheric blocking state with positive anomalies in the three $\mathrm{SH}$ blocking regions. Taken over the entire reanalysis period the FEM-BVVARX cluster states are positive and negative blocking phases. Summer-averaged transition states for both periods resemble the southern annular mode. Taken as a whole, these results indicate that there has been a fundamental change in the regime states of the $\mathrm{SH}$ atmospheric circulation whereby the negative blocking pattern has 

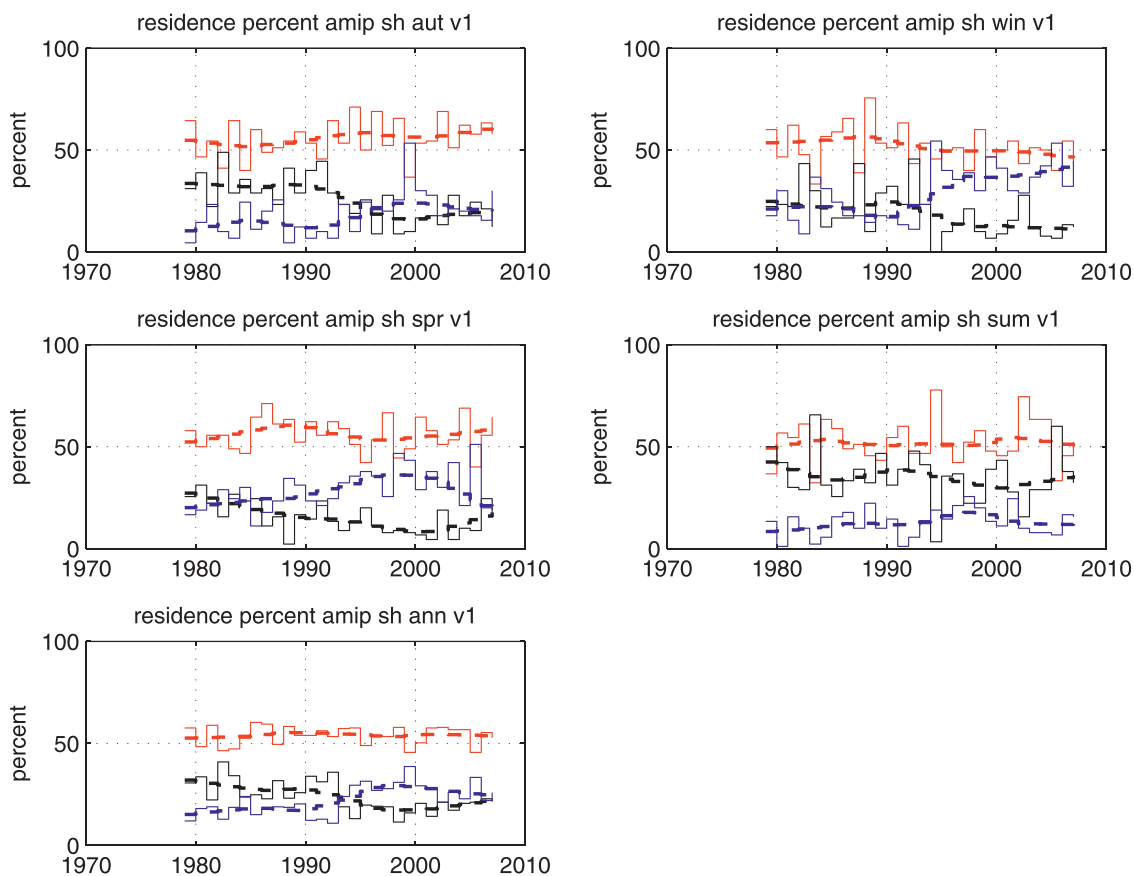

FIG. 19. FEM-BV-VARX percentage of time resident in each given state corresponding to Figs. 16 and 18 for AMIP 500 hPa 1979-2008: positive blocking state 1 (blue; see Fig. 18, top left), negative blocking state 2 (black; see Fig. 18, top right), and the transition state (red; see Fig. 18, bottom). Persistency parameter set to 0 . The dashed lines are a LOESS fit to the timeaveraged data. The solid lines are the values and averaging periods of the data.

been progressively over time replaced by the SAM as a metastable state and that the SAM is intensifying while blocking is in decline, both in terms of occurrence and persistency.

Comparison of cluster affiliation sequences (Viterbi paths) to split-flow blocking indices, commonly used in operational meteorology and to construct blocking climatologies, was found to show qualitative agreement. Closer inspection revealed significant differences between the BoM index and FEM-BV-VARX composite states, both hemispheric and for the Australian region. The BoM index composite states were found to be representative of split flow, while the FEM-BV-VARX composites capture localized coherent anomalies associated with blocking. For the Australian region, the Tibaldi index and criteria states were found to be closely matched to those using the BoM index with positive anomalies to the southeast of New Zealand and at higher latitudes. FEM-BV-VARX
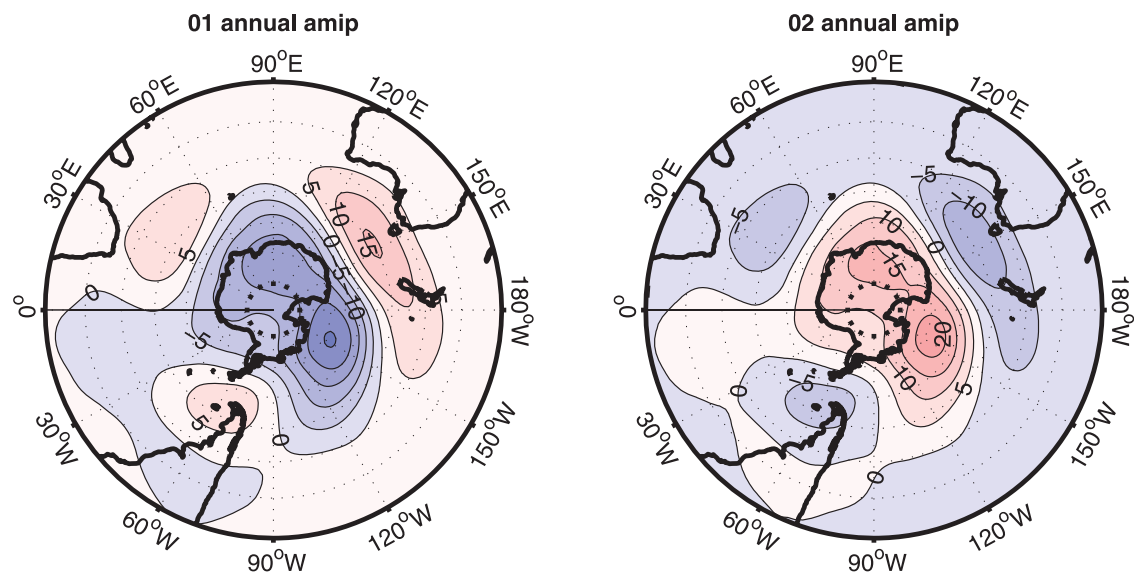

FIG. 20. Composites using the Viterbi paths shown in Figs. 17 and 19 for AMIP $500 \mathrm{hPa}$ 1979-2008: persistency parameter set to 100. 

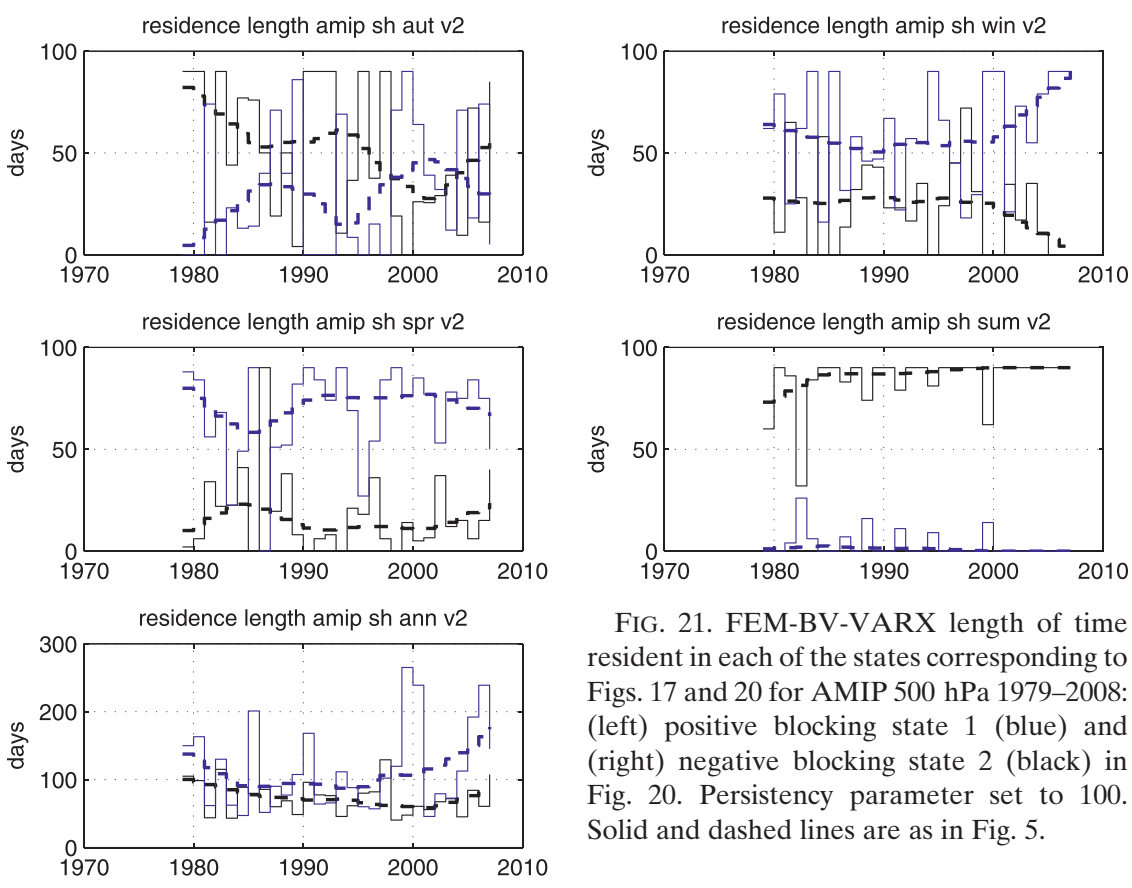

FIG. 21. FEM-BV-VARX length of time resident in each of the states corresponding to Figs. 17 and 20 for AMIP $500 \mathrm{hPa}$ 1979-2008: (left) positive blocking state 1 (blue) and (right) negative blocking state 2 (black) in Fig. 20. Persistency parameter set to 100 . Solid and dashed lines are as in Fig. 5.

composites were found to have positive anomalies in the midlatitudes between Australia and New Zealand in regions where blocks are on average most typically found to occur.

Analysis of AMIP model data shows little evidence of a strong SAM-like metastable state but that the system transitions between positive and negative blocking states, with almost no evidence of a transition state, and that this holds regardless of choices of smoothing parameter. The model also reveals a strong tendency to lock into either state for extended periods (several months) at a time, preferring the positive blocking phase in winter and spring and the negative blocking phase in summer. The model has an observable trend toward longer residence periods in the positive blocking state, particularly post-1994. The conclusion is that SST changes alone are not capable of inducing a transition to the SAM as a metastable state.

It seems tempting to infer through comparison of the NCEP and climatologically forced AMIP datasets that changes in greenhouse gas and ozone forcing over the latter half of this century (present in the NCEP data but not in the AMIP model) act to modify the existing tendency for declining blocking via a transition of the negative blocking state toward the SAM. Presumably the seasonal nature of ozone forcing is responsible for imposing the strong seasonality toward spring/summer as the trending months (Polvani et al. 2011). The role of intrinsic processes (ENSO, MJO, etc.) and external factors such as radiative forcing is clearly central to understanding the

drivers of the observed changes in the SH circulation. In a companion study, we apply the full VARX-FEM to identify and quantify the dominant external factors driving changes to the metastability of the Southern Hemisphere.

Acknowledgments. This work was possible thanks to financial assistance provided by a CSIRO Complex Systems Science Networks Conference Travel Support grant and additional funding through ACCESS: The Australian Community Climate and Earth-System Simulator effort, CSIRO's Wealth from Oceans and Climate Adaptation Flagships. This study is part of the British Antarctic Survey Polar Science for Planet Earth Programme. It was partly funded by the Natural Environment Research Council. The development of the Adaptive FEM-BV-VARX used in this paper was funded by Swiss National Research Foundation Grant 200021_131845 “AnaGraph.”

\section{REFERENCES}

Baines, P., 1983: A survey of blocking mechanisms, with application to the Australian region. Aust. Meteor. Mag., 31, 27-36.

Berner, J., and G. Branstator, 2007: Linear and nonlinear signatures in the planetary wave dynamics of an AGCM: Probability density functions. J. Atmos. Sci., 64, 117-136.

Branstator, G., and J. Berner, 2005: Linear and nonlinear signatures in the planetary wave dynamics of an AGCM: Phase space tendencies. J. Atmos. Sci., 62, 1792-1811.

Charney, J., and J. DeVore, 1979: Multiple flow equilibria in the atmosphere and blocking. J. Atmos. Sci., 36, 1205-1216. 
Cheng, X., and J. Wallace, 1993: Cluster analysis of the Northern Hemisphere wintertime 500-hpa height field: Spatial patterns. J. Atmos. Sci., 50, 2674-2696.

Collins, W. J., and Coauthors, 2008: Evaluation of the HadGEM2 model. Hadley Centre Tech. Note 74, 47 pp.

Corti, S., F. Molteni, and T. Palmer, 1999: Signature of recent climate change in frequencies of natural atmospheric circulation regimes. Nature, 398, 799-802.

Crommelin, D. T., and E. Vanden-Eijnden, 2006: Fitting timeseries by continuous-time Markov chains: A quadratic programming approach. J. Comput. Phys., 217, 782-805.

Franzke, C., 2009: Multi-scale analysis of teleconnection indices: Climate noise and nonlinear trends. Nonlinear Processes Geophys., 16, 65-76.

—, D. T. Crommelin, A. Fischer, and A. J. Majda, 2008: A hidden Markov model perspective on regimes and metastability in atmospheric flows. J. Climate, 21, 1740-1757.

—, I. Horenko, A. J. Majda, and R. Klein, 2009: Systematic metastable atmospheric regime identification in an AGCM. J. Atmos. Sci., 66, 1997-2012.

Frederiksen, J. S., 1982: A unified three-dimensional instability theory of the onset of blocking and cyclogenesis. J. Atmos. Sci., 39, 969-982.

— 1983: A unified three-dimensional instability theory of the onset of blocking and cyclogenesis. II: Teleconnection patterns. J. Atmos. Sci., 40, 2593-2609.

— eling. Entropy, 10, 635-683.

,-- , and M. J. Zidikheri, 2012: Stochastic subgrid parameterizations for atmospheric and oceanic flows. Phys. Scr., 85, 068202, doi:10.1088/0031-8949/85/06/068202.

Gates, W. L., 1992: AMIP: The Atmospheric Model Intercomparison Project. Bull. Amer. Meteor. Soc., 73, 1962-1970.

Horenko, I., 2008: On simultaneous data-based dimension reduction and hidden phase identification. J. Atmos. Sci., 65, 1941-1954.

_ 2009: On robust estimation of low-frequency variability trends in discrete Markovian sequences of atmospheric circulation patterns. J. Atmos. Sci., 66, 1941-1954.

_ 2010a: Finite element approach to clustering of multidimensional time series. SIAM J. Sci. Comput., 32, 62-83.

- , 2010b: On clustering of non-stationary meteorological time series. Dyn. Atmos. Oceans, 49, 164-187.

- , 2010c: On the identification of nonstationary factor models and their application to atmospheric data sets. J. Atmos. Sci., 67, 1559-1574.

— , 2011: Nonstationarity in multifactor models of discrete jump processes, memory, and application to cloud modeling. J. Atmos. Sci., 68, 1493-1506.

Jones, D., and I. Simmonds, 1994: A climatology of Southern Hemisphere anticyclones. Climate Dyn., 10, 333-348.

Majda, A., C. Franzke, A. Fischer, and D. Crommelin, 2006: Distinct atmospheric regimes despite nearly Gaussian statistics:
A paradigm model. Proc. Natl. Acad. Sci. USA, 103, 83098314.

Martin, G. M., M. A. Ringer, V. D. Pope, A. Jones, C. Dearden, and T. J. Hinton, 2006: The physical properties of the atmosphere in the new Hadley Centre Global Environmental Model (HadGEM1). Part I: Model description and global climatology. J. Climate, 19, 1274-1301.

Noar, P., 1983: Numerical modelling of blocking, with reference to June 1982. Aust. Meteor. Mag., 31, 37-49.

O'Kane, T., and J. Frederiksen, 2008: A comparison of statistical dynamical and ensemble prediction methods during blocking. J. Atmos. Sci., 65, 426-446.

Polvani, L. M., D. W. Waugh, G. J. P. Correa, and S.-W. Son, 2011: Stratospheric ozone depletion: The main driver of twentiethcentury atmospheric circulation changes in the Southern Hemisphere. J. Climate, 24, 795-812.

Pook, M., and T. Gibson, 1999: Atmospheric blocking and storm tracks during SOP-1 of the FROST Project. Aust. Meteor. Mag., 1, 51-60.

Rashid, H., M. Dix, and A. Hirst, 2009: Surface energy balance in the ACCESS models: Comparisons with observation based flux products. CAWCR Res. Lett., 3 (12), 34-42.

Risbey, J., M. Pook, P. McIntosh, M. Wheeler, and H. Hendon, 2009: On the remote drivers of rainfall variability in Australia. Mon. Wea. Rev., 137, 3233-3253.

Shakina, N., and A. Ivanova, 2010: The blocking anticyclones: The state of studies and forecasting. Russ. Meteor. Hydrol., 35, 721730.

Sinclair, M., 1996: A climatology of anticyclones and blocking in the Southern Hemisphere. Mon. Wea. Rev., 124, 245-263.

Thompson, D., and S. Solomon, 2002: Interpretation of recent Southern Hemisphere climate change. Science, 296, 895-899.

Tibaldi, S., 1993: Low-frequency variability and blocking as diagnostic tools for global climate models. Proc. NATO Advanced Research Workshop on Prediction of Interannual Climate Variations, Trieste, Italy, NATO-ASI, 173-182.

_ , and F. Molteni, 1990: On the operational predictability of winter blocking. Tellus, 42A, 343-365.

_ , E. Tosi, A. Navarra, and L. Pedulli, 1994: Northern and Southern Hemisphere seasonal variability of blocking frequency and predictability. Mon. Wea. Rev., 122, 1971-2003.

Trenberth, K., and K. Mo, 1985: Blocking in the Southern Hemisphere. Mon. Wea. Rev., 113, 3-21.

Wright, A. D. F., 1974: Blocking action in the Australian region. Australia Bureau of Meteorology Tech. Rep. 10, 27 pp.

Wright, W., 1994: Seasonal climate summary southern hemisphere (autumn 1993): A second mature ENSO phase. Aust. Meteor. Mag., 43, 205-221.

Zidikheri, M., J. Frederiksen, and T. O'Kane, 2007: Multiple equilibria and midlatitude atmospheric blocking: A re-examination. Frontiers in Turbulence and Coherent Structures, World Scientific Lecture Notes in Complex Systems, Vol. 6, World Scientific, 59-86. 